\title{
Educação em saúde: vivência de enfermeiros da Estratégia Saúde da Família no interior de Pernambuco
}

\author{
Health education: experience of nurses in the Family Health Strategy in Pernambuco \\ Educación en salud: experiencia de enfermeras de la Estrategia de Salud Familiar en \\ Pernambuco
}

Yris Luana Rodrigues da Silva ${ }^{1}$, Juliana Monteiro Costa ${ }^{2}$.

\begin{abstract}
RESUMO
Objetivo: Discutir a atuação do enfermeiro acerca das práticas educativas na Estratégia de Saúde da Família. Métodos: Estudo descritivo de abordagem qualitativa. A coleta dos dados foi realizada por meio de entrevistas semiestruturadas. Para a análise das entrevistas, foi utilizada a técnica Análise de conteúdo na modalidade Análise temática. Resultados: A partir da análise do conteúdo das entrevistas emergiram quatro categorias: Desenvolvimento das ações educativas na perspectiva dos enfermeiros/Planejamento das ações educativas pelos enfermeiros/Metodologias utilizadas nas práticas educativas/Potencialidades e fragilidades na execução das Ações Educativas em Saúde. Conclusão: A educação em saúde é ferramenta utilizada pelos enfermeiros para auxiliar na prevenção de doenças, com metodologias pautadas na transmissão de conhecimento, a fim de favorecer mudanças de comportamento do indivíduo e/ou da população. É necessário que os gestores garantam condições para a execução das atividades educativas nas ESF através da disponibilização de recursos materiais, investimento em estrutura física das unidades de saúde e ações de educação permanente aos enfermeiros da ESF.
\end{abstract}

Palavras-chave: Atenção primária em saúde, Educação em saúde, Enfermeiro.

\section{ABSTRACT}

Objective: To discuss the role of nurses regarding educational practices in the Family Health Strategy. Methods: A descriptive study with a qualitative approach. Data collection was carried out through semistructured interviews. For the analysis of the interviews, the technique of Content Analysis was used in the Thematic Analysis modality. Results: From the analysis of the content of the interviews, four categories emerged: Development of educational actions from the perspective of nurses / Planning of educational actions by nurses / Methodologies used in educational practices / Potentials and weaknesses in the execution of Educational Actions in Health. Conclusion: Health education is a tool used by nurses to assist in disease prevention, with methodologies based on the transmission of knowledge, in order to favor changes in the behavior of the individual and / or the population. It is necessary for managers to guarantee conditions for carrying out educational activities in the ESF through the provision of material resources, investment in the physical structure of the health units and to promote permanent education actions for the ESF nurses.

Keywords: Primary health care, Health education, Nurse.

\section{RESUMEN}

Objetivo: Discutir el papel de lo enfermero sobre las prácticas educativas en la estrategia de salud familiar. Métodos: Estudio descriptivo con enfoque cualitativo. La recolección de datos se realizó mediante entrevistas semiestructuradas. Para análisis de las entrevistas, se utilizó la técnica de análisis de contenido en la modalidad de análisis temática. Resultados: Del análisis del contenido de las entrevistas surgieron cuatro categorías: Desarrollo de acciones educativas desde la perspectiva de las enfermeras/Planificación de acciones educativas por parte de los enfermeros/Metodologías utilizadas en prácticas educativas/Potenciales y debilidades en la implementación de acciones educativas en salud. Conclusión: La educación en salud es una herramienta utilizada por las enfermeras para ayudar en la prevención de enfermedades, con

1 Escola Técnica Estadual Arlindo Ferreira dos Santos, Sertânia - PE. *E-mail: yrisluana1990@hotmail.com

2 Faculdade Pernambucana de Saúde (FPS), Recife - PE.

SUBMETIDO EM: 6/2020

ACEITO EM: 7/2020

PUBLICADO EM: 9/2020

REAS/EJCH | Vol.12(12) | e4277 | DOI: https://doi.org/10.25248/reas.e4277.2020 Página 1 de 8 
metodologías basadas en la transmisión de conocimiento, a fin de favorecer cambios en el comportamiento del individuo y/o la población. Es necesario que los gerentes garanticen las condiciones para la ejecución de actividades educativas en la ESF mediante la provisión de recursos materiales, la inversión en la estructura física de las unidades de salud y las acciones de educación permanente para las enfermeras de la ESF.

Palabras clave: Atención primaria de salud, Educación en salud, Enfermeros.

\section{INTRODUÇÃO}

Há 40 anos, a Declaração de Alma-Ata identificou a saúde primária como uma estratégia central para a meta de "saúde para todos", além disso, abordou a organização dos sistemas de saúde. Desde então, os valores e princípios da educação primária e dos cuidados com a saúde, incluem o direito à saúde, a equidade, a solidariedade, a justiça social, a participação e a ação multissetorial, dentre outros (ETIENNE CF, 2018).

O Sistema Único de Saúde (SUS) foi implementado no início dos anos noventa e, logo depois, os municípios assumiram um papel de liderança para a sua expansão. A Atenção Primária em Saúde (APS) tem sido central para fortalecimento do SUS, particularmente por meio da Estratégia Saúde da Família (ESF), introduzida pela Política Nacional de Cuidado.

A organização do processo de trabalho na APS deve priorizar a garantia da universalidade do acesso, integralidade da atenção e melhoria do bem-estar do usuário, além das condições de trabalho da equipe. Ademais, necessita de profissionais com uma ampliação do seu núcleo de saberes, que além da competência técnica, desenvolvam dimensões políticas e de gestão do trabalho em saúde (MASSUDA A, et al., 2018; GALAVOTE HS, et al., 2016).

Nesse contexto a A ESF surge, como política pública que pressupõe práticas e princípios da APS, tendo como objetivo principal o de materializar o fazer e o pensar em saúde, através de um modelo assistencial que enfoca a lógica de intervenção territorial. A ESF trabalha com o desafio de conduzir a mudança do modelo assistencial através do trabalho em equipe multiprofissional, capaz de produzir cuidado integral e longitudinal para a população pertencente ao seu território. Além disso, utiliza tecnologias complexas, porém menos densas e centrada nas relações sociais, como é o caso da educação em saúde (ALMEIDA ER, et al., 2016).

As práticas educativas compõem o trabalho na área da saúde, porém, frequentemente, percebe-se que tais ações não são priorizadas nas fases de planejamento, organização, gestão e execução. Com a expansão da medicina preventiva, na década de 40 , algumas regiões brasileiras receberam ações educativas com ênfase em estratégias autoritárias e com caráter biomédico, a população era apenas receptora de informações (FALKENBERG MB, et al., 2014).

A educação em saúde é considerada um instrumento que tem como objetivo principal a conscientização e a liberdade do indivíduo. Entretanto, em seu processo de configuração, houve uma forte influência dos modelos que não priorizavam o indivíduo, mas a doença e a transmissão de conhecimento do profissional para o paciente, assumindo uma estrutura rígida e tradicional. A atual estruturação e organização da saúde no Brasil traz a ampliação dessa concepção, ao possibilitar ganho de objetivos teóricos centrados na autonomia, além de práticas inovadoras e diferenciadas de educação em saúde na atenção primária (SILVA ALQ, et al., 2013).

Assim, no contexto da atenção primária no SUS, a educação em saúde é inserida como uma atividade que deve ser desenvolvida com a participação e a responsabilidade de todos os profissionais que compõem a equipe de saúde nos serviços de assistência à saúde. Essa prática, portanto, assume um importante papel na afirmação e no fortalecimento dos princípios do SUS, ao proporcionar um contato direto com os usuários, garantindo um mecanismo de interlocução entre gestão, profissionais e usuários de saúde (MARTINS RAS e SOUZA CA, 2017; BRASIL, 2012).

O objetivo desse estudo é compreender a vivência de enfermeiros no desenvolvimento das ações de Educação em Saúde na ESF. 


\section{MÉTODOS}

Trata-se de uma pesquisa de natureza qualitativa, realizada em um município localizado no Sertão de Pernambuco, nove ESF compõem a rede de Atenção Primária. A população do estudo foi composta por 8 enfermeiros que atuavam nas ESF do município.

Foram incluídos no estudo: enfermeiros de ambos os sexos, com vínculo empregatício temporário ou efetivo. Após o aceite da participação da entrevista, os profissionais assinaram o Termo de Consentimento Livre e Esclarecido (TCLE). Foram excluídos da pesquisa os profissionais que estivessem de férias ou de licença médica por algum motivo de saúde.

Os dados foram coletados nas unidades da ESF e o instrumento utilizado foi uma entrevista sem dirigida, organizada a partir de um roteiro previamente elaborado. Os dados sociodemográficos dos participantes foram coletados, as entrevistas foram realizadas individualmente no consultório de Enfermagem da instituição de saúde, com horário previamente acordado. Os participantes foram informados quanto aos objetivos do estudo e a garantia de sigilo das gravações e anonimato. Para isso, no decorrer da apresentação, os entrevistados serão denominados Enf. 1, Enf. 2 e assim por diante.

Os dados foram analisados através da técnica de Análise Temática proposta por Minayo, onde através dela é possível encontrar respostas para as questões formuladas e confirmar ou não as afirmações estabelecidas antes do trabalho de investigação (hipóteses). Além disso, outra função diz respeito à descoberta do que está por trás dos conteúdos manifestos, indo além das aparências do que está sendo comunicado (FONTANELLA BJ, et al., 2008).

Neste estudo foram preservados os preceitos éticos estabelecidos pela Resolução 510/16 do Conselho Nacional de Saúde (BRASIL, 2016). A coleta dos dados só teve início após assinatura da carta de Anuência pela da Secretária de Saúde de Sertânia e aprovação do projeto de pesquisa pelo Comitê de Ética em Pesquisa com Seres Humanos da Faculdade Pernambucana de Saúde (CEP/FPS) através do CAAE número 02317418.0.0000.5569 e Parecer número 3.033.526.

Os riscos relacionados à participação no estudo foram mínimos. Não obstante, as pesquisadoras ficaram atentas e, caso acontecesse desconforto e/ou constrangimento por parte dos participantes, as mesmas abririam um espaço de acolhimento aos mesmos.

\section{RESULTADOS E DISCUSSÃO}

Foram entrevistados oito enfermeiros da ESF, seis do sexo feminino, as idades variaram entre 28 e 52 anos, cuja média foi de 38,3 anos. Seis participantes eram casados e dois não possuíam filhos, quatro se graduaram em universidades públicas e os demais em instituições privadas. Todos os enfermeiros cursaram alguma pós-graduação latu senso, três na área de saúde pública e dois com mestrado em educação. Cinco profissionais eram efetivos e três contratados temporários. Cinco entrevistados tinham mais de um vínculo profissional, todos eram cristãos e relataram experiência em outro nível de atenção.

\section{Desenvolvimento das ações de educação em saúde na perspectiva dos enfermeiros da Estratégia Saúde da Família (ESF)}

Na primeira categoria, os participantes relataram como executam e desenvolvem as ações de educação em saúde nas ESF. Os enfermeiros ressaltaram a importância do trabalho em equipe, buscando engajar cada membro da ESF em todas as etapas do processo de desenvolvimento das atividades educativas, conforme pode ser observado nas falas a seguir:

"Desenvolvo com os Agentes de Saúde [...], técnico de enfermagem, médico e com a população." (Enf 3, 28 anos)

"Eu desenvolvo as ações, primeiramente, nas reuniões de equipe [...] Eles escolhem o tema de saúde, veem alguns temas [...] Nessa reunião, discutimos e resolvemos qual tema será trabalhado, algumas vezes trabalhamos mais de um tema, junto com médico, dentista e os ACS." (Enf 2, 37 anos) 
"Nós aqui trabalhamos em equipe [...] A gente normalmente divide as tarefas com o enfermeiro, médico, odontólogo, os ACS, auxiliar de saúde bucal e a técnica de enfermagem [...] Cada um fala da sua vivência em cada palestra que a gente realiza e nós gostamos muito de envolver a população nas nossas palestras." (Enf 7, 52 anos)

Estudo realizado em Santa Catarina, constatou que a variedade de profissionais que compõem uma equipe de saúde amplia o planejamento da assistência e facilita o acesso da população à profissionais de áreas e conhecimentos diversos (ANDRADE ACV, et al., 2013). É importante que o enfermeiro delegue e engaje toda equipe na execução das ações educativas, para evitar a sobrecarga de atividades e a priorização de atividades burocráticas e assistenciais, conforme pontuado pelos enfermeiros abaixo:

"Sempre procuro fazer uma palestra, abordar um tema, colocar até o ACS também no meio, a técnica de enfermagem, a dentista, o médico, para não ficar só no lado do enfermeiro [...] Eu sempre coloco a equipe para fazer." (Enf 1, 40 anos)

"Primeiro porque, isso [atividade educativa] sempre cai pra o enfermeiro da unidade. Então, vai muito da minha vontade de querer fazer." (Enf 8, 28 anos)

"Muitas dão certo. O único problema é quando é muita palestra, sem ter alguma atividade que você mostre pra essas pessoas, porque eles não querem só ouvir eles não querem. Eles vem, mas querem atendimento." (Enf 3, 28 anos)

Estudo realizado por Ramos CFV,et al.(2018) com dezessete enfermeiros do Acre, apontou a relevância do envolvimento da equipe multiprofissional no planejamento e efetivação das práticas educativas. $O$ estudo sinalizou que a demanda excessiva de consultas, a priorização o atendimento ambulatorial e a valorização da produtividade dificultam a realização das atividades educativas.

Nessa perspectiva, a pesquisa realizada no Crato-CE realçou que a atuação desses profissionais tornase fragmentada devido ao acúmulo de atividades o que contribui para essa abordagem curativa (OLIVEIRA MB, et al., 2018).

\section{Planejamento das ações educativas pelos enfermeiros da ESF}

$\mathrm{Na}$ segunda categoria, os participantes descreveram como é realizado o planejamento das ações educativas que serão executadas na ESF. Os enfermeiros realizam o planejamento em equipe, nas reuniões mensais da ESF. Nessa reunião, são definidas as datas e os temas a serem abordados na execução das atividades educativas. Além disso, são definidos quais profissionais irão executar as atividades daquele mês. Como pode ser observado nas falas abaixo:

"Sempre faço reuniões mensais com os ACS e nessas reuniões a gente vê o que pode fazer naquele mês." (Enf 1, 42 anos)

"Nas reuniões de equipe eu planejo conforme o que eu tenho disponível." (Enf 2, 37 anos)

"Nas reuniões com a equipe, a gente determina quais são os temas que vamos trabalhar no mês, então a gente agenda um dia, determina o público alvo e a gente determina da equipe quem vai participar: o médico, a dentista, o enfermeiro, os agentes de saúde." (Enf 5, 41 anos)

Em um estudo com oito enfermeiros das ESF, realizado no Rio Grande do Sul, os resultados corroboraram com nosso estudo, pois os resultados apontaram o planejamento como um instrumento importante e necessário para a execução das práticas educativas na atenção primária, por possibilitar maior resolutividade sobre a saúde dos usuários e maior impacto nos indicadores de saúde da população (SILOCCHI C e JUNGES $J R, 2017)$. Dos entrevistados, apenas dois enfermeiros realizam o planejamento das atividades educativas através das demandas identificadas pela equipe multiprofissional:

"O planejamento é feito praticamente com a demanda." (Enf 4, 34 anos) 
"A gente convoca pra uma reunião e cada um do médico ao auxiliar de serviços gerais da sua opinião e daí a gente tira, um consolidado, e ver quais os pontos mais importantes que venham beneficiar a população." (Enf 7, 52 anos)

Estudo realizado por Roecker S, et al. (2013), encontrou resultados divergentes, pois a maioria dos entrevistados utilizou o levantamento do perfil demográfico, social e epidemiológico, além dos diálogos com usuários como base para a definição das prioridades das ações educativas.

O estudo concluiu ainda que as práticas educativas deveriam ser planejadas após a realização do diagnóstico das necessidades da população, levando em consideração os determinantes do processo saúdedoença, para assim refletirem positivamente nas condições de saúde dos usuários, família e comunidade.

\section{Estratégias utilizadas nas ações de educação em saúde}

$\mathrm{Na}$ terceira categoria, os entrevistados elencaram as abordagens utilizadas nas ações de educação em saúde na ESF. O uso de palestras, banners e panfletos foram trazidos como os principais métodos utilizados nas atividades educativas do serviço de saúde, conforme descritos a seguir:

"A metodologia é palestra [...] Sinceramente, sinceramente eu fico perdido nessa questão, porque eu não vejo outra forma, porém a forma que a gente faz eu não vejo muito resultado." (Enf 8, 28 anos)

"A gente faz a palestra e depois eles tiram dúvidas." (Enf 5, 40 anos)

"Geralmente aqui a gente usa palestra, slides, banners e panfletos... panfletagem. E principalmente a palestra." (Enf 3, 28 anos)

Estudo realizado na Bahia (BA) corrobora com os resultados da presente pesquisa, pois os entrevistados fizeram uso apenas da palestra nas ações de educação em saúde. Além disso, o estudo identificou a ênfase no modelo de transmissão do conhecimento e modelo biomédico da assistência à saúde. Estudo de Revisão que abordou as diferentes concepções acerca da Promoção da Saúde consolidou que a população tem sido vista como receptora de informações, sem capacidade para trazer contribuições, experiências e propostas para o cuidado com a saúde (BOMFIM ESB, et al., 2017; MORAES MCL, 2017).

Alguns enfermeiros destacaram a importância de envolver o usuário nas ações de educação em saúde, tornando-o participante ativo no processo educativo. Os entrevistados destacaram, também, que a utilização de rodas de conversas pode proporcionar a valorização dos saberes dos usuários e uma maior interação entre profissionais e população, além da mudança do modelo de transmissão de conhecimentos, onde o profissional é único detentor do saber. Nesse sentido, a atividade educativa pode gerar mudanças de comportamento na comunidade, promover saúde e garantir o protagonismo do sujeito. As falas a seguir ilustram bem este aspecto:

"Eu acredito que o ideal seria uma roda de conversa [...] Porque na palestra, você chega lá na frente, só conversa [...]Você não escuta a experiência [...] O profissional é dono do saber e ninguém mais sabe." (Enf 4, 38 anos)

"Usamos esse tipo de conversação [...] Porque a gente acha muito importante envolver a população [...] Nesse sistema a gente vê que o êxito é melhor que a palestra." (Enf 7, 52 anos)

"O público não quer só ouvir, eles não querem apenas ouvir, querem algo que mostre pra eles o que eles estão ouvindo." (Enf 3, 28 anos)

Estudo semelhante realizado em Belo Horizonte - MG, com profissionais da ESF, com o objetivo de compreender como as práticas são realizadas neste ambiente, evidenciou que a educação em saúde concretiza-se como um exercício de autonomia e favorece o processo de construção da cidadania, quando se utiliza da escuta, da problematização e da produção conjunta do conhecimento, pois proporciona a expressão do cidadão nas diferentes dimensões da saúde e do autocuidado (GAZZINELLI MF, et al., 2015). 


\section{Potencialidades e Fragilidades na execução das ações de educação em saúde na perspectiva dos enfermeiros}

Na quarta categoria os enfermeiros apontaram os pontos fortes em suas práticas educativas de saúde, tais como: a troca de experiências na unidade de saúde através da problematização e da valorização do conhecimento prévio dos usuários, como também a promoção de ações com resultados positivos na comunidade com a participação dos Agentes Comunitários de Saúde (ACS).

"Gosto muito de abordar essa parte investigativa, trabalhar com essa partezinha da bagagem anterior deles sobre esse assunto, problematizar, trocas de experiências e assim vai [... ]" (Enf 6, 42 anos)

"A gente tem também uma atividade na comunidade, ACS em Ação, eu planejo com eles (Agentes de Saúde) e a gente escolhe um tema [...] Aí faz uma roda de conversa, num local de sombra, numa árvore [...] A população senta, uns perguntam, e é bem interessante." (Enf 2, 37 anos)

Estudo realizado com oito enfermeiros no estado da Paraíba demonstrou que apesar das dificuldades e desafios, pode-se inferir que a ESF é um cenário que facilita ações intersetoriais. Não obstante, torna-se necessário que os profissionais tenham um novo olhar diante dos entraves e das ações de promoção da saúde para construir novos saberes. Em adição a isso, estudo realizado com enfermeiros da ESF do Ceará, identificou a importância da utilização dos espaços públicos na comunidade para realização das atividades educativas (OLIVEIRA MB, et al., 2018; TRIGUEIRO JS, et al., 2009). Nas falas a seguir os entrevistados relatam os desafios:

"O ambiente físico e a questão de materiais [...] Eu sinto falta de ter materiais [...] Faltam, as vezes, alguns materiais de suporte como: impressora, TV pra passar alguma coisa, o projetor que também não tinha." (Enf 2, 37 anos)

"O que eu acho mais complicado é a estrutura física [...] Realmente a gente não tem estrutura física." (Enf 4, 34 anos)

"Essa questão de insumos, de panfletos e também de divulgação são os principais entraves." (Enf 5, 40 anos)

Estudo realizado no Paraná encontrou diversos entraves enfrentados pelos enfermeiros na execução das ações educativas, dentre elas: a insuficiência de recursos físicos, materiais e financeiros. O estudo verificou, ainda, que o profissional deve buscar alternativas para realização e priorização da prática educativa, não apenas como atividade, mas como prática necessária para reorientação da Atenção Primária em Saúde (ROECKER S, et al., 2012). Os enfermeiros e a população geralmente priorizam as abordagens clínicas e o modelo biomédico de atenção à saúde, as falas abaixo ilustram essa realidade:

"A demanda dificulta, porque se tivesse uma quantidade menor eu poderia toda semana utilizar um dia só para trabalhar educação em saúde [...] Mas se eu tiro um dia de atendimento, já complica [...]A burocracia é muito grande." (Enf 1, 42 anos)

"Eles (usuários) vêm, mas querem atendimento [...] Eu não paro o atendimento da unidade. Eu faço o atendimento, o médico faz o dele, a gente não para o atendimento." (Enf 3, 28 anos)

"Eu tenho uma área muito grande aqui com dois pontos de apoio [...] Se eu tiro um dia para palestra, uma horinha que seja, já é o tempo que eu deixo de atender." (Enf 9, 28 anos)

Estudo realizado no Rio de Janeiro evidenciou que as práticas de cuidados dos enfermeiros estão voltadas aos aspectos biológicos. Dessa maneira, o cuidado expressivo não é fortalecido, o que pode comprometer a integralidade do cuidado à saúde. Esse olhar advém do avanço tecnológico que tem contribuído para um cuidado baseado nas em procedimentos técnico-científicos e forte influência do modelo hegemônico tradicional (ACIOLI S, et al., 2014; SERRANO MPT, et al., 2011). 
A educação permanente é elemento essencial para que o profissional de saúde tenha embasamento teórico e se sinta encorajado para realizar suas práticas educativas de forma efetiva e que possam impactar na saúde dos usuários. A falta de capacitação e treinamento foram apontadas como lacunas pelos enfermeiros, conforme descrito abaixo:

"Capacitação, treinamento, atualização [...] Porque é muito complicado você chegar e dizer: "faça isso!", sem ao menos Ihe dar condição de trabalho." (Enf 4, 34 anos)

"Primeiro a falta de capacitação geral de todos os profissionais [...] Se você não é capacitado, você não tem aquele estímulo pra fazer." (Enf 8, 28 anos)

A Educação Permanente em Saúde (EPS) é uma forma de aprendizagem no trabalho, neste cenário, os profissionais aprendem e ensinam, baseia-se na aprendizagem significativa e na possibilidade de ressignificação das práticas profissionais do seu cotidiano. Apresenta como objetivos: gerar reflexão sobre o processo de trabalho, autogestão, mudança institucional e transformação das práticas. Estudo realizado em Goiânia-GO constatou que a EPS permite ao profissional capacitado planejar, desenvolver e avaliar ações que atendam às necessidades da população (SERRANO MTP, et al., 2011; PAULINO VCP, et al., 2012).

A pesquisa registra como limitação a impossibilidade de generalização dos seus resultados para toda a população, já que o mesmo foi realizado em ESF de um único município de Pernambuco representando características e relações existentes em âmbito local. Outra limitação verificada é o fato de este estudo não ter incluído os demais profissionais pertencentes a equipe da ESF. Diante disso, os resultados podem ser utilizados como base para identificação das principais dificuldades e com isso, nortear a elaboração de estratégias e políticas de enfrentamento para os problemas apresentados.

\section{CONCLUSÃO}

Os resultados apontam que ações educativas de saúde são geralmente executadas sem o conhecimento dos indicadores do território pertencente à ESF, com predomínio no uso de palestras. Tal fato ocorre, principalmente pela sobrecarga de atividades do profissional enfermeiro, escassez de recursos materiais e ausência de capacitação profissional. Observa-se ainda que a participação multiprofissional nas ações educativas de saúde evita a sobrecarga de atividades do profissional enfermeiro, além de proporcionar aos usuários diferentes saberes acerca de um tema específico. Por fim, a educação continuada e o incentivo por parte dos gestores públicos são fundamentais para que as ações educativas possam ser exitosas e reflitam nos profissionais através da valorização dos saberes dos usuários e compreensão do contexto social e cultural em que estão inseridos.

\section{REFERÊNCIAS}

1. ACIOLI S, et al. Práticas de cuidado: o papel do enfermeiro na atenção básica. Rev enferm UERJ, 2014;22(5):63742.

2. ALMEIDA ER, et al. Family health nurses'teaching practice in the health education development. Interface, 2016; 20(57):389-401.

3. ANDRADE ACV, et al. Planejamento das ações educativas pela equipe multiprofissional da Estratégia Saúde da Família.Mundo da Saúde, 2013, 37(4):439-449.

4. BOMFIM ESB, et al. Atuação do Enfermeiro acerca das práticas educativas na Estratégia de Saúde da Família. Rev enferm UFPE on line, 2017;11 (Supl. 3):1398-1402.

5. BRASIL. Conselho Nacional de Saúde. Resolução no 510, de 7 de abril de 2016. Diário Oficial da União: Seção 1. p. 44-46.

6. BRASIL. Política Nacional de Atenção Básica. Secretaria de Atenção à Saúde. Departamento de Atenção Básica. Brasília, 2012.

7. BRASIL. Política Nacional de Educação Permanente em Saúde: o que se tem produzido para o seu fortalecimento? Brasília, 2018.

8. ETIENNE CF. Promoting universal health through primary health care. Rev Panam Salud Publica, 2018;42(1): 145.

9. FALKENBERG MB, et al. Educação em saúde e educação na saúde: conceitos e implicações para a saúde coletiva. Ciência \& Saúde Coletiva, 2014;19(3):847-852. 
10. FONTANELLA BJ, RICAS J \&Turato ER. Amostragem por saturação em pesquisas qualitativas em saúde: contribuições teóricas. Cad. Saúde Pública, 2008; 24(1): 17-27.

11. GALAVOTE, HS et al. O trabalho do enfermeiro na atenção primária à saúde. Esc Anna Nery, 2016;20(1):90-98.

12. GAZZINELLI MF, et al. Práticas educativas grupais na atenção básica: padrões de interação entre profissionais, usuários e conhecimento. Rev Esc Enferm USP,2015; 49(2):284-291.

13. MARTINS RAS, SOUZA CA. A educação em saúde no contexto da atenção primária em saúde. REFACS, 2017;5(Supl 2):282-288.

14. MASSUDA A, et al. Remembering Alma-Ata: challenges and innovations in primaryhealth Care in a middle-income city in Latin America. Rev Panam Salud Publica, 2018;42:1-09.

15. MINAYO MCS. O Desafio do Conhecimento: Pesquisa Qualitativa em Saúde. São Paulo: HUCITEC; 2007.

16. MINAYO MCS. Pesquisa Social. Teoria, método e criatividade. Petrópolis: Vozes; 2001.

17. MORAES MCL.Promoção da saúde: visitando conceitos e ideias. REFACS,2017; 5(1):75-79.

18. OLIVEIRA MB, et al. Educação em saúde como prática de enfermeiros na estratégia saúde da família. Rev Rene, 2018; 14(5):894-903.

19. PAULINO VCP, et al. Educação permanente e saúde da família. Rev. enferm. UERJ, 2012; 20(3):312-316.

20. RAMOS CFV, et al. Education practices: research-action with nurses of Family Health Strategy. Rev Bras Enferm, 2018;71(3):1144-1151.

21. ROECKER S, et al. O trabalho educativo do enfermeiro na estratégia saúde da família. Texto Contexto Enferm, 2013;22(1):157-165.

22. ROECKER S, et al. Trabalho educativo do enfermeiro na Estratégia Saúde da Família: dificuldades e perspectivas de mudanças. Rev Esc Enferm USP,2012; 46(3):641-649.

23. SERRANO MTP, et al. Cuidar em enfermagem: como desenvolver a(s) competência(s). Revista de Enfermagem Referência, 2011; 3(3): 15-23.

24. SILOCCHI C, JUNGES JR. Equipes de atenção primária: dificuldades no cuidado de pessoas com doenças crônicas não transmissíveis. Trab. Educ. Saúde, 2017, 15(2):599-615.

25. SILVA ALQ, et al. Práticas educativas mais utilizadas pelos enfermeiros da atenção básica: Uma revisão bibliográfica. Revista Científica do ITPAC, 2013; 6(4):01-05

26. TRIGUEIRO JS, et al. Percepção de enfermeiros na educação em saúde na tuberculose. Cienc Cuid Saude, 2009;8(4):660-666.

27. TURATO ER. Métodos qualitativos e quantitativos na área da saúde: definições, diferenças e seus objetos de pesquisa. Revista de Saúde Pública, 2005; 39(3): 507-514. 